\title{
TRIGGERING THE RADIO EMISSION FROM AE AQR
}

\author{
D. STEEGHS ${ }^{1}$, J. KUIJPERS ${ }^{1}$, L. FLETCHER ${ }^{1}$ \\ M. ABADA-SIMON ${ }^{1}$, K. HORNE ${ }^{2}$, G. RAMSAY ${ }^{1}$ \\ 1. Astronomical Institute, Utrecht University, The Netherlands \\ 2. Physics \& Astronomy, University of St.Andrews, UK
}

\begin{abstract}
We propose a model for the acceleration of particles by magnetic pumping to drive the radio emission from AE Aqr.
\end{abstract}

\section{Introduction}

The peculiar CV AE Aqr displays irregular, flare-like radio emission which varies in intensity and duration (minutes to hours). Even during the interflare periods there is usually some quiescent radio flux (Abada-Simon et al. 1993). The flare-like emission is attributed to synchrotron emission from high energy electrons in expanding plasma clouds (Bastian, Dulk \& Chanmugam 1988). We propose that magnetic pumping (betatron acceleration) accelerates these electrons.

As the white dwarf rotates, the magnetic field strength is periodically varied as it opens and closes around the accreting diamagnetic 'blobs' and the secondary star, which lies within the light cylinder (Fig. 1). As the magnetic pressure experienced by the accreting blobs changes by a factor of $\sim 4$ every rotation, internal shocks are generated and surface plasma instabilities arising from the relative velocity of the blob and the field disrupt and strip off the surface layers. This results in the loading onto the oscillating field of particles which can be energized by magnetic pumping.

\section{Magnetic pumping}

In a changing magnetic field $p_{\perp}^{2} / B$ is conserved, so that the particle perpendicular momentum, $p_{\perp}$, increases as the field increases. If some momentum can be transferred, by particle scattering, to the parallel direction (not affected by the field change) the particle energy increases with time, $t$, as 

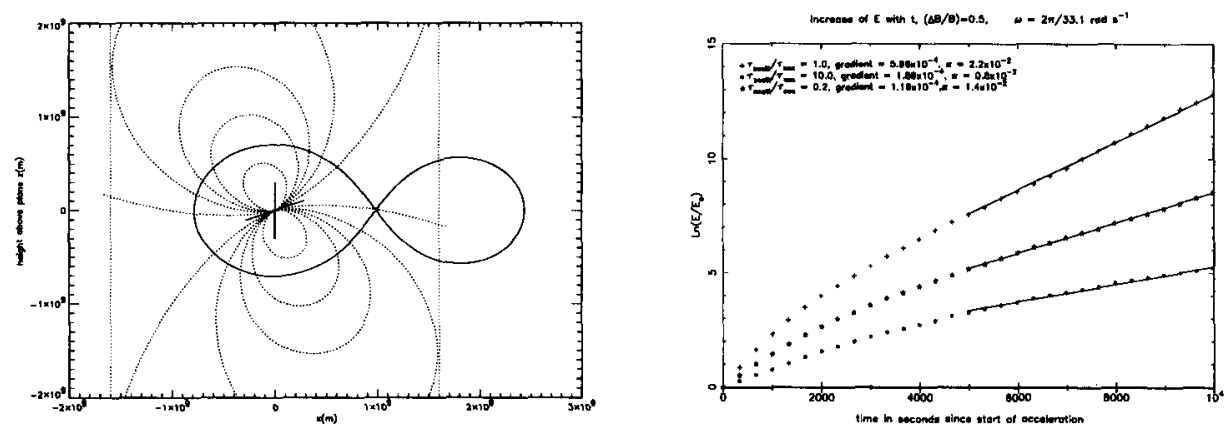

Figure 1. Left panel: side view of the binary system, showing the tilted dipole field and the position of the light-cylinder. Right panel: a particle simulation result, showing the energy increase due to magnetic pumping. (Kuijpers et al. 1996)

$(\Delta B / B)^{2} \exp (\alpha t)$ (at relativistic energies), where $\alpha$ is a parameter which maximizes when the particle collision time equals the field oscillation time (white dwarf rotation period). Particle simulations of pumping (Kuijpers et al. 1996) show that standing oscillations accelerate more efficiently than traveling Alfvén waves. We calculate values for $\alpha$ and find that, under realistic conditions, enough particles to account for the flare energy can be accelerated up to the required energies in a few thousand seconds, even when energy losses (e.g. synchrotron) are included.

\section{Radio emission}

The particles are trapped by magnetic mirroring in the converging white dwarf magnetic field lines. Fresh material is continually supplied by accretion and, as acceleration continues, the loading of the field increases. When the total kinetic energy of the particles becomes comparable with the total energy of the magnetic field, the field no longer contains them and a MHD instability is triggered resulting in a catastrophic loss of particle confinement and the ejection of a plasmoid, which produces the enhanced radio emission. The magnetosphere can then be energized again for the next flare. The quiescent radio emission is attributed to the synchrotron emission of the fast particles as they are accelerated between eruptions.

\section{References}

Abada-Simon, M., Lecacheaux, A., Bastian, T.S., et al., 1993, Ap. J., 406, 692

Bastian, T.S., Dulk, G.S., Chanmugam, G., 1988, Ap. J., 324, 431

Kuijpers J., Fletcher L., Abada-Simon M., et al., 1996, in preparation 